SELECTED PAPER AT NCSP'19

\title{
Flexible Edge Component Detection Using Image Power Spectrum Sparsity
}

\author{
Naw Jacklin Nyunt, Yosuke Sugiura and Tetsuya Shimamura \\ Graduate School of Science and Engineering, Saitama University \\ Shimo-Okubo 255, Sakura-ku, Saitama 338-8570, Japan \\ E-mail: \{jacklin, sugiura, shima\}@ sie.ics.saitama-u.ac.jp
}

\begin{abstract}
A novel method for edge component detection based on image power spectrum sparsity is presented. The edge size can be varied by changing the block size and threshold parameter to obtain the desired edge component. The image is first divided into sub-blocks and the power spectrum sparsity for each sub-block is calculated. On the basis of the image power spectrum sparsity value, each block is verified by the threshold value to determine the edge component. The experimental results show that the proposed method is suitable for object tracking because of the novel feature of the flexible edge size, which can dramatically reduce the amount of data to be stored.
\end{abstract}

\section{Introduction}

Image edge detection is a fundamental tool that plays an important role in image processing, machine vision and computer vision. The image edge comprises the sharp changes in brightness that make up the discontinuities of the image. The discontinuities and changes describe image boundaries that reveal the important features of the image. Image edge detection dramatically reduces the amount of information to store as it filters out useless information and preserves important features useful for image segmentation, object recognition and object tracking. A number of edge detectors based on a single derivative such as the Roberts operator, Sobel operator, Prewitt operator and Canny operator have been developed by various researchers [1]. There are also edge detectors based on a second derivative, such as the Laplacian operator and Laplacian of Gaussian (LOG) operator. Among these edge detectors, the Canny operator is the optimal operator that can detect true edge points with the minimum error. The Canny operator guarantees edge detection with three performance criteria: (i) good detection, (ii) good localization and (iii) only one response to a single edge [3]. Edge detection algorithms based on artificial neural networks are also being developed to fulfill the increasing requirements of the accuracy of algorithms in image processing fields [4].

All of the edge detection algorithms mentioned above can be used to detect an image edge with a fixed edge size. How- ever, for object tracking, which does not require all of the detailed edge part, an edge detection algorithm with a flexible edge size is necessary. The use of a flexible edge size method enables the amount of data to be stored to be dramatically reduced. Thus, in this paper, we propose a flexible edge component detection algorithm. The basic idea of the proposed method is to detect the edge component block by block in the frequency domain using the image power spectrum sparsity by changing the block size and threshold parameter. The aim of this paper is (i) to introduce a novel edge detection component method in which a novel concept named image power spectrum sparsity is used and (ii) to show that the edge size is flexible so that the amount of data to be stored is significantly reduced.

The paper is organized as follows. Section 2 describes details of the proposed method. Section 3 discusses the experimental results and conclusions are given in Section 4.

\section{Proposed Method}

In our proposed method, we measure the edge component block by block. Firstly, the input image $x(u, v)$ with the size of $256 \times 256$ is divided into $n \times n$ sub-blocks, $x_{(j, k)}(u, v)$ where $(j, k=1,2, \ldots, n)$, with the desired window size $(M=2,4,8$ and $n=256 / M)$. Each sub-block $x_{(j, k)}(u, v)$ is transformed into the frequency domain, $X_{(j, k)}(u, v)$, by fast Fourier transform (FFT). The power spectrum for each sub-block is calculated as

$$
P_{(j, k)}(u, v)=\left|X_{(j, k)}(u, v)\right|^{2}
$$

Figure 1 shows (a) the input image divided into $64 \times 64$ subblocks with the window size of $M=4$ and (b) power spectrum of each sub-block in (a).

\subsection{Power spectrum sparsity}

After determining the power spectrum for each sub-block, it is necessary to define the edge component for each image power spectrum sub-block. In Fig. 1(b), it can be seen that the edge component of the power spectrum sub-block has more image frequencies than a flat component, which does not have 


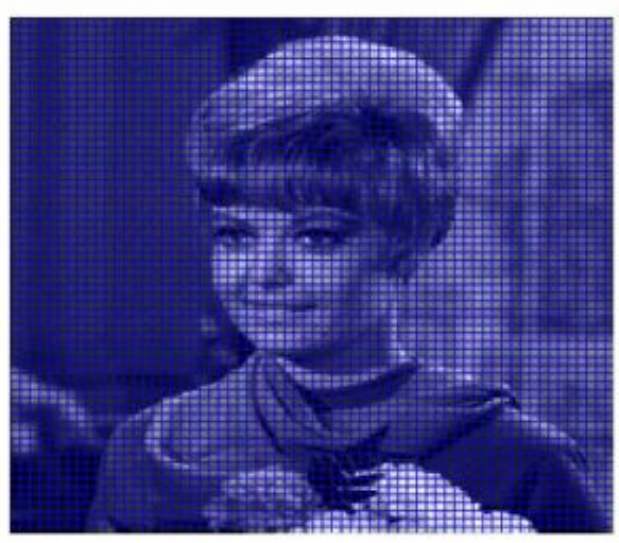

(a)

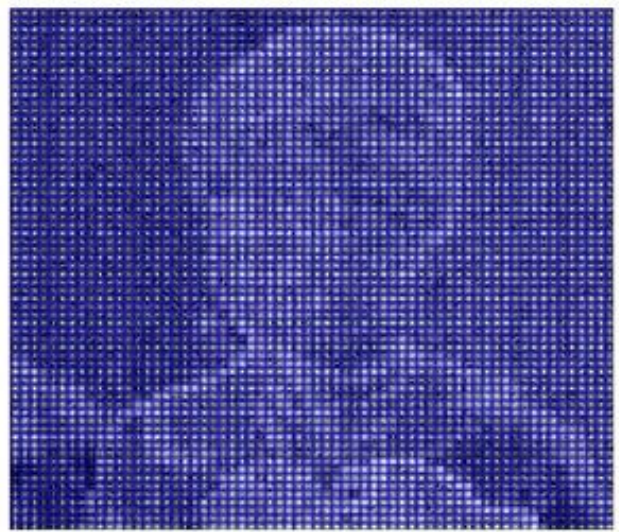

(b)

Figure 1: (a) Input image divided into blocks $(M=4)$, (b) Power spectrum of each block in (a)

any image edge component. Therefore, by estimating the number of image frequencies contained in the sub-block, the image edge can be calculated. To obtain the number of frequencies contained in the sub-block, the idea of power spectrum sparsity, $S$, introduced in [2] is applied. The power spectrum sparsity for each block is calculated as

$$
S_{(j, k)}(u, v)=\frac{P_{D(j, k)}(u, v)}{P_{D h(j, k)}(u, v)+P_{D v(j, k)}(u, v)}
$$

where $P_{D(j, k)}(u, v), P_{D h(j, k)}(u, v)$ and $P_{D v(j, k)}(u, v)$ represent the sum of the whole sub-block region, the horizontal region and the vertical region of the image power spectrum of the corresponding sub-block, respectively, as shown in Fig. 2. The image power spectrum sparsity $S$ gives the number of image frequencies contained in the image. For the image sub-block, $S$ is maximum when it contains no image frequency components in the horizontal or vertical region of the image power spectrum sub-block or when the image subblock is flat with the same texture [2]. In other words, when

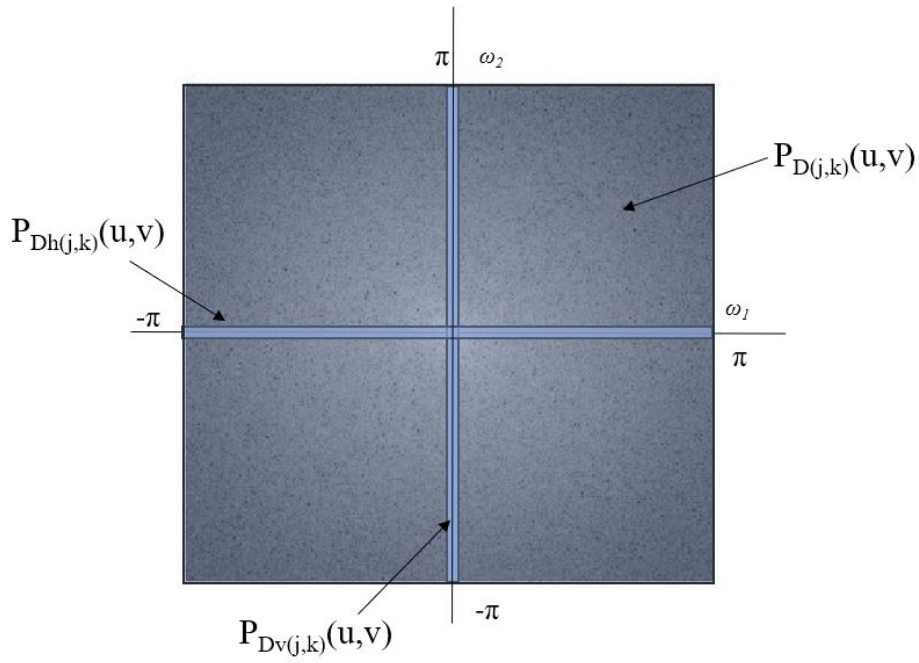

Figure 2: Power spectrum sparsity of image sub-block

the image frequencies are more concentrated in the horizontal and vertical regions of the image power spectrum of the subblock (i.e., when the image sub-block contains higher image frequencies), then $S$ will be smaller. Therefore, we can detect the image edge region by choosing the smallest $S$ value of the whole image. Figure 3 shows (a) the image power spectrum for each sub-block with the window size $M=16$ and (b) $S$ for each sub-block in (a). The red sub-blocks in Fig. 3 show some of the sub-blocks with high image frequencies. It can be seen that the sub-block with the smaller $S$ represents the edge component of the image. Therefore, the edge component can be detected on the basis of $S$.

\subsection{Edge component determination by thresholding}

In order to determine the edge component for the whole image, a simple threshold is calculated as

$$
T H=t \times \min S
$$

where $\min S$ represents the smallest $S$ value of the image sub-block for the whole image. $t$ is the threshold parameter, which is selected in accordance with the image type. The image edge component is obtained as

$$
x_{(j, k)}(u, v)= \begin{cases}\operatorname{Max}(x(u, v)), & S_{(j, k)}(u, v) \leq T H \\ 255-\operatorname{Max}(x(u, v)), & S_{(j, k)}(u, v)>T H\end{cases}
$$

A sub-block whose $S$ value is less than or equal to $T H$ is defined as the edge component by assigning the maximum intensity value, and a sub-block whose $S$ value is greater than $T H$ is defined as the non edge component by subtracting the maximum intensity value from 255 . Finally, the image edge component is obtained. 


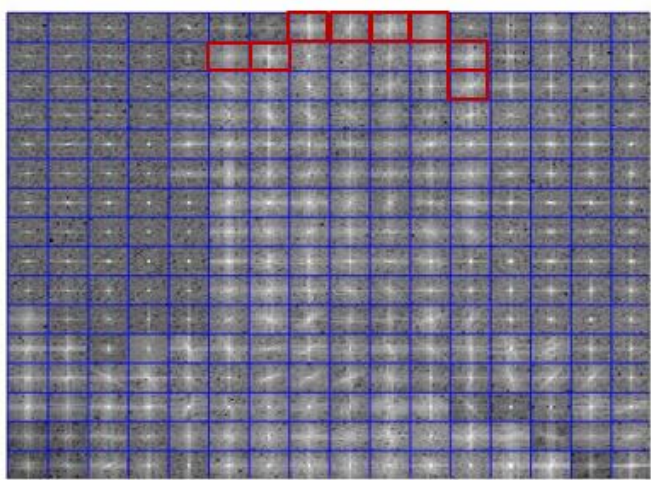

(a)

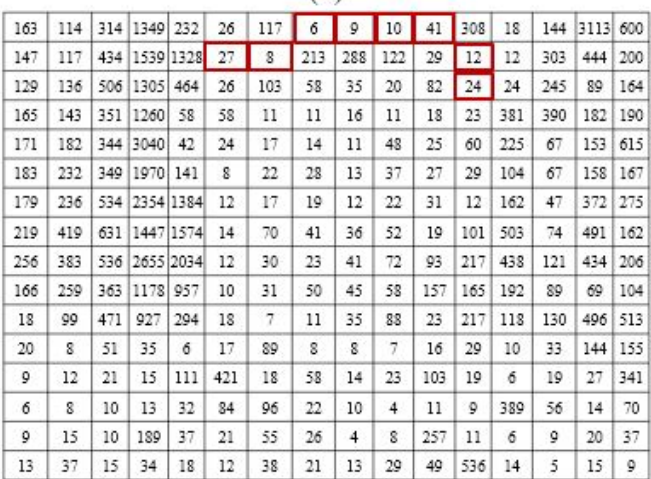

(b)

Figure 3: (a) Image power spectrum with window size $M=$ 16, (b) Power spectrum sparsity value for each sub-block

\subsection{Diagram of proposed method}

The input image $(256 \times 256)$ with the block size $M=$ $2,4,8$ and the threshold parameter $t$ is obtained. The input image is first divided into sub-blocks with the input block size. Each sub-block is transformed into the frequency domain by fast Fourier Transform(FFT). Then, the power spectrum for each sub-block is calculated with Eq. (1). To obtain the number of image frequencies contained in each sub-block, the image power spectrum sparsity is calculated with Eq. (2). To determine the image edge component, Eq. (3) is calculated with the input threshold parameter. Finally, the image edge component is obtained using Eq. (4). This method is summarized in Fig. 4.

\section{Experimental Results}

To show the performance of the proposed method, it is compared with the Sobel operator, Prewitt operator and Canny operator. Figure 5 shows the performances of edge detection for the image GIRL with the image size $256 \times 256$. The results show that the Sobel and Prewitt operators do not detect some of the edge parts, whereas the Canny operator detect all of the image edge parts. Figure 5(e) shows the re-

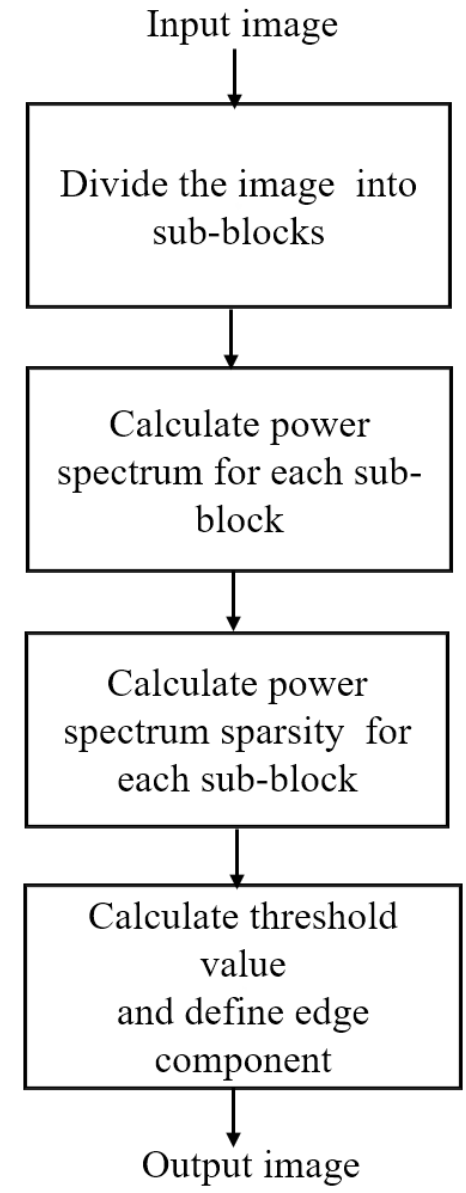

Figure 4: Block diagram of proposed method

sults of the proposed method with the window size $M=2$ and the threshold parameter $t=40$. It can be seen that the proposed method can detect the edge components that the Sobel and Prewitt operators do not detect. The Canny operator detects all of the edge components but the edge size is fixed. To show the benefit of our proposed method, the results for different window sizes are also shown in Figs. 5(f) and $5(\mathrm{~g})$, in which the window sizes and the threshold parameters are $M=4, t=20$ and $M=8, t=15$, respectively. A larger window size needs a smaller threshold parameter to determine the image edge component. By looking at image edge components for different window sizes, it is seen that the proposed method can dramatically reduce the amount of information to be stored. The important image edge component can be detected by changing the threshold parameter in accordance with the image type.

\section{Conclusion}

A flexible edge component detection method in which im- 


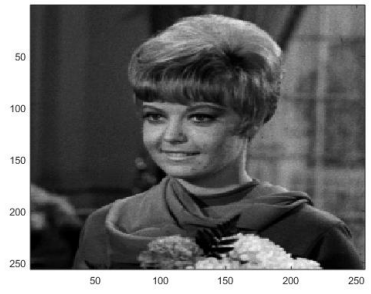

(a)Original image

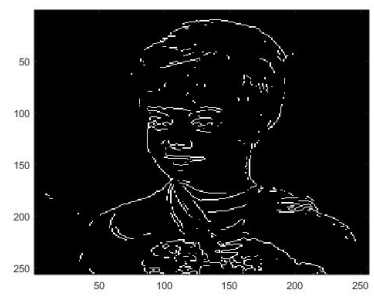

(c)Prewitt

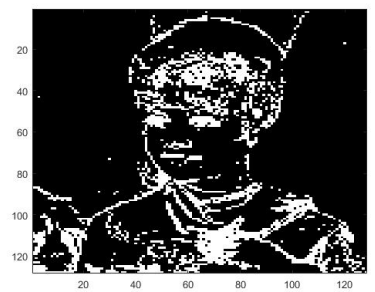

(e)Proposed $(M=2, t=40)$

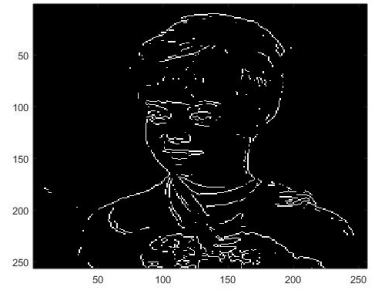

(b)Sobel

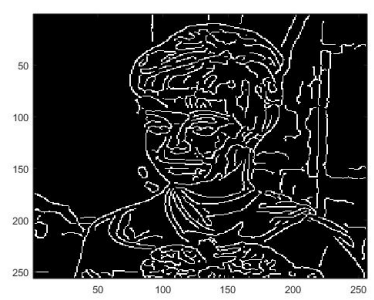

(d)Canny

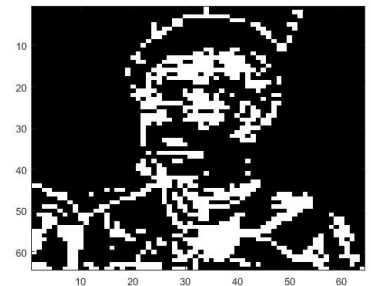

(f)Proposed ( $M=4, t=20)$

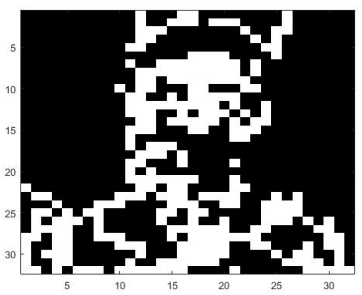

(g)Proposed $(M=8, t=10)$

Figure 5: Results of various methods for the image GIRL

age power spectrum sparsity is used was proposed. The block size is flexible and the threshold parameter is also adjustable in accordance with different applications. The amount of data to be stored can be significantly reduced and the important edge component of the image can be detected by the proposed method. The proposed method is suitable for edge component detection, and is applicable for object tracking where a detailed edge is not necessary.

\section{References}

[1] T. Acharya and A. K. Ray: Image Processing Principles and Applications, John Wiley and Sons, Inc., 2005.

[2] N. J. Nyunt, Y. Sugiura and T. Shimamura: Parametric
Wiener filter based on image power spectrum sparsity, Journal of Signal Processing, Vol. 22, No.6, pp. 287-297, 2018.

[3] J. Canny: A computational approach to edge detection, IEEE Transactions on Pattern Analysis and Machine Intelligence, Vol. PAMI-8, No.6, pp. 679-698, 1986.

[4] J. Gu, Y. Pan and H. Wang: Research on the improvement of image edge detection algorithm based on artificial neural network, Optik 126, pp. 2974-2978, 2015. 